\title{
Insights into Proximal Pouch Dilatation Following Adjustable Gastric Banding-a Form of Inattentional Blindness?
}

\author{
John B. Dixon • Ben J. Dixon • Christopher S Cobourn
}

Published online: 15 May 2012

(C) Springer Science+Business Media, LLC 2012

Proximal gastric pouch dilatation (PPD) or enlargement is the most common cause for revision surgery, and a leading cause of band intolerance and failure following laparoscopic adjustable gastric band surgery. We have recently reported some interesting, unexpected, and clinically important factors associated with the development of PPD in 3,000 consecutive cases of primary laparoscopic adjustable gastric banding (LAGB) procedures. These associations were clear and highly statistically significant, but not perceived by the bariatric team managing these patients. Is this a case of inattentional blindness in clinical practice? Inattentional blindness is the failure to recognize a visual stimulus that is present within your visual field. This phenomenon becomes more apparent when higher levels of concentration are required to complete a task as attention is focussed on the task itself rather than on unexpected findings or alternative explanations.

The first analysis examined the effect of oesophageal hiatal repair at the time of band placement and the subsequent need for surgical revision for PPD [1]. Analysis demonstrated that repair of the esophageal hiatus was associated with an increased risk of PPD, with an adjusted odds

\footnotetext{
J. B. Dixon $(\bowtie)$

Vascular \& Hypertension Division-Obesity Research,

Baker IDI Heart \& Diabetes Institute,

Melbourne, Australia

e-mail: john.dixon@bakeridi.edu.au
}

\section{B. J. Dixon}

Department of Surgery, St Vincents Hospital,

University of Melbourne,

Melbourne, Australia

C. S. Cobourn

Surgical Weight Loss Centre,

Mississauga, ON, Canada ratio of 2.3 (95\% CI, 1.4-3.8, $p<0.001$ ). Exploration and repair rates increased throughout the series from 27 to $60 \%$ and $90 \%$ in consecutive groups of 1,000 patients, on the expectation that this additional step would reduce the risk of a significant hiatal defect being missed, with the concern that an oversight may increase the risk of subsequent surgical revision [2]. We examined the revision rate for PPD during the first 36 months for all patients who had completed at least 3 years follow-up after band placement. This analysis demonstrated a significant and sustained reduction in the incidence of PPD with time, but this was not related to greater rate of crural repair. The increased risk of subsequent revision with exploration and repair was most prominent early in the series, but at no stage did exploration and repair reduce risk. The staff in the surgical practice now believe that the reduced incidence of PPD is more likely related to a better understanding of the mechanism of action of the band over time by both staff and patients. There is now a focus on band adjustments for satiety and satisfaction rather than restriction. The practice of repairing significant hiatal hernia and careful exploration in patients with significant gastrooesophageal reflux symptoms appears reasonable, but exploration of a normal esophageal hiatal area in an asymptomatic patient is not supported by this large carefully documented series.

A second clinically important observation was that women and lower age were independently associated with an increased risk of PPD requiring revision [3]. Exploring the data proved interesting as the age effect was restricted to women with younger women at a higher risk, and men and post menopausal women at much lower risk. As all cases of PPD were classified by barium study into symmetrical and asymmetrical dilatations, we examined for any influence on type of dilatation. Asymmetrical dilatations, found to occur earlier with more acute symptoms, dominated in younger 
women. As true gastric prolapse of stomach from below to above the band was very unusual at revision, asymmetrical dilatation is presumably related to greater stretching or distensability of the anterior wall of the proximal stomach under the influence of pressure generated in the esophagus during periods of excessive restriction or obstruction. Given the risk of PPD and the type encountered in younger women, we hypothesise that sex hormones, in particular progesterone, may contribute to the distensability of gastric smooth muscle. Clinically, increased restriction is often reported by women during pregnancy and the pre-menstrual phase of their cycle. It is important to understand that these observations may not be restricted to LAGB surgery and could be important in retaining the anatomical integrity of all forms of bariatric surgery which have a small gastric pouch intrinsic to the mechanism of action.

The clinical implications of these findings are clear. For oesophageal hiatal exploration and repair "if it ain't broke don't fix it" fits nicely with "first do no harm". While we need to learn more about the mechanism involved in the PPD risk of younger women, we can provide guidance by reinforcing the importance of satisfaction and satiety rather than "restriction" $[4,5]$. The most beneficial advice involves talking about food choice, chewing and allowing time for food to clear the band, and never over adjusting the band or allowing a patient to feel the band is tight. Clinicians should be alert and responsive to premenstrual and pregnancy band tightness, and teach all patients the signs and symptoms of excessive band tightness and when to seek help to prevent pressure-related PPD.

The perception of our clinical practice and its outcomes is influenced by our experience. This "experience" is a complex and continually evolving blend of traditional teaching, personal analysis, peer opinion and reactions to previous critical events or adverse outcomes. Although these factors may play a powerful role in determining our actions, they are prone to bias and are rarely evidence based. Undue attention on a particular factor may occur when it is prominently reported by peers at scientific meetings and in the literature. We tend to place little emphasis on information that we do not expect to be important. In fact, evidence suggests we may not even perceive these events at all. When focused on a particular task, one may miss the gorilla in our midst [6].

Inattentional or perceptive blindness is important in surgery and surgical practice. In the immediate or usual context, it is highly relevant to the complexity and advances in surgical equipment and techniques. The more assistance one has to focus on the immediate surgical task, the greater the likelihood of not detecting the unexpected. Considering and addressing inattentional blindness in the surgical area will be a major focus of developments in surgery as it has been in aviation and other industries. We here describe a chronic form of perceptual blindness where signals regarding patient outcomes are clear but are not seen. As most bariatric surgical patients are women, there has been a failure to recognize that they are overrepresented in those requiring revisional surgery. And there was an acceptance that crural exploration and repair would reduce revision rates. Careful collection and timely examination of data will allow this form of blindness to be addressed, and development of surgical registries provides but one method of addressing perceptual (inattentional) blindness.

Conflict of Interest $\mathrm{CC}$ is the Director of the surgical practice attended by the patient cohort described in this publication. JD acts as a consultant for Allergan Inc. and Metagenics Inc and is on the Medical Advisory Board for Nestle Australia. He was previously a member of the Scientific Advisory Board and received research funding from Scientific Intake Inc. JD currently receives a Research Fellowship from the National Health and Medical Research Council of Australia and also receives research support from Allergan Inc. CC's practice currently receives research support from Allergan Inc, and CC has previously received speaker and consultancy fees from Allergan Inc. BD has no conflicts to declare.

\section{References}

1. Dixon JB, Cobourn CS. Exploration of esophageal hiatus: does crural repair reduce proximal pouch distension? Surg Obes Relat Dis. 2012. doi:10.1016/j.soard.2011.12.015.

2. Gulkarov I, Wetterau M, Ren CJ, et al. Hiatal hernia repair at the initial laparoscopic adjustable gastric band operation reduces the need for reoperation. Surg Endosc. 2008;22(4):1035-41.

3. Dixon JB, Cobourn CS. Risk of gastric pouch enlargement with adjustable gastric banding in premenopausal women: sex hormones may play a role? Ann Surg. 2012. doi:10.1097/SLA.0b01e3182504665.

4. Burton PR, Brown WA. The mechanism of weight loss with laparoscopic adjustable gastric banding: induction of satiety not restriction. Int J Obes (Lond). 2011;35 Suppl 3:S26-30.

5. Dixon AF, Dixon JB, O'Brien P. Laparoscopic adjustable gastric banding induces prolonged satiety: a randomised blind crossover study. J Clin Endocrinol Metab. 2005;90(2):813-9.

6. Simons DJ, Chabris CF. Gorillas in our midst: sustained inattentional blindness for dynamic events. Perception. 1999;28(9):105974 . 